\title{
Modeling Infectious Diseases Dynamics: Dengue Fever, a Case Study
}

\author{
Maíra Aguiar ${ }^{1}$, Nico Stollenwerk ${ }^{1}$ and Bob W. Kooi ${ }^{2}$ \\ ${ }^{1}$ Centro de Matemática e Aplicações Fundamentais, Lisbon University \\ ${ }^{2}$ Faculty of Earth and Life Sciences, Department of Theoretical Biology, Vrije Universiteit \\ ${ }^{1}$ Portugal \\ ${ }^{2}$ The Netherlands
}

\section{Introduction}

Throughout human history, infectious diseases have caused debilitation and premature death to large portions of the human population, leading to serious social-economic concerns. Many factors have contributed to the persistence and increase in the occurrence of infectious disease (demographic factors, political, social and economic changes, environmental change, public health care and infrastructure, microbial adaptation, etc.), which according to the World Health Organization $(\mathrm{WHO})$, are the second leading cause of death globally ( $\approx 23 \%$ of deaths) after cardiovascular diseases (WHO, 2010).

Research on basic and applied aspects of host, pathogen, and environmental factors that influence disease emergence, transmission and spread have been supported so far, and the development of diagnostics, vaccines, and therapeutics has been greatly increased. In recent years, mathematical modeling became an interesting tool for the understanding of infectious diseases epidemiology and dynamics, leading to great advances in providing tools for identifying possible approaches to control, including vaccination programs, and for assessing the potential impact of different intervention measures.

Epidemiological models are a formal framework to convey ideas about the components of a host-parasite interaction and can act as a tool to predict, understand and develop strategies to control the spread of infectious diseases by helping to understand the behaviour of the system under various conditions. They can also aid data collection, data and interpretation and parameter estimation. The purpose of epidemiological models is to take different aspects of the disease as inputs and to make predictions about the numbers of infected and susceptible people over time as output.

In the early $20^{\text {th }}$ century, mathematical models were introduced into infectious disease epidemiology, and a series of deterministic compartment models such as SI (susceptible-infected), SIS (susceptible-infected-susceptible), and e.g SIR (susceptibleinfected-recovered) have been proposed based on the flow patterns between compartments of hosts. In our days, most of the models developed try to incorporate other factors focusing on several different aspects of the disease, which can imply rich dynamic behaviour even in the most basic dynamical models. Factors that can go into the models include the duration 
of disease, the duration of infectivity, the infection rate, the waning immunity, and so forth. In such a way, differential equation models are a simplified representation of reality, which are designed to facilitate prediction and calculation of rates of change as functions of the conditions or the components of the system.

There are two common approaches in modeling, the deterministic and the stochastic one. In the first case, the model is one in which the variable states are uniquely determined by parameters in the model and by sets of previous states of these variables. In mathematics, a deterministic system is a system in which no randomness is involved in the development of future states of the system. In a stochastic model, randomness is present, and variable states are not described by unique values, but rather by probability distributions. Stochastic epidemic models are appropriate stochastic processes that can be used to model disease propagation. Disease propagation is an inherently stochastic phenomenon and there are a number of reasons why one should use stochastic models to capture the transmission process. Real life epidemics, in the absence of intervention from outside, can either go extinct with a limited number of individuals getting ultimately infected, or end up with a significant proportion of the population having contracted the disease in question. It is only stochastic, as opposed to deterministic, models that can capture this behavior and the probability of each event taking place.

Only few stochastic processes can be solved explicitly. The simplest and most thoroughly studied stochastic model of epidemics are based on the assumption of homogeneous mixing, i.e. individuals interact randomly at a certain rate. The mean field approximation is a good approximation to be used in order to understand better the behavior of the stochastic systems in certain parameter regions, where the dynamics of the mean quantities are approximated by neglecting correlations, giving closed ordinary differential equations (ODE) systems, hence mathematically deterministic systems which are easier to analyze.

In the following section of this chapter we present the properties of the basic SIR epidemic model for infectious diseases with a summary of the analysis of the dynamics, identifying the thresholds and equilibrium points. The goal is to introduce notation, terminology, and results that will be generalized in later sections on more advanced models motivated by dengue fever epidemiology as an example of multi-strain systems.

\section{The SIR epidemic model}

The SIR epidemic model divides the population into three classes: susceptible $(S)$, Infected $(I)$ and Recovered $(R)$. It can be applied to infectious diseases where waning immunity can happen, and assuming that the transmission of the disease is contagious from person to person, the susceptibles become infected and infectious, are cured and become recovered. After a waning immunity period, the recovered individual can become susceptible again. This model was for the first time proposed by William Ogilvy Kermack and Anderson Gray McKendrick in 1927 (Weisstein, 2010). The model was brought back to prominence after decades of neglect by Anderson and May (Anderson \& May, 1979).

In the simple SIR epidemics without strain structure of the pathogens we have the following reaction scheme for the possible transitions from one to another disease related state, 
susceptibles $S$, infected $I$ and recovered $R$,

$$
\begin{aligned}
S+I \stackrel{\beta}{\longrightarrow} I+I \\
I \stackrel{\gamma}{\longrightarrow} R \\
R \stackrel{\alpha}{\longrightarrow} S
\end{aligned}
$$

for a host population of $N$ individuals, with contact and infection rate $\beta$, recovery rate $\gamma$ and waning immunity rate $\alpha$. The dynamic model in terms of ordinary differential equations (ODE) reads,

$$
\begin{aligned}
& \dot{S}=-\frac{\beta}{N} I S+\alpha(N-S-I) \\
& \dot{I}=\frac{\beta}{N} I S-\gamma I,
\end{aligned}
$$

where we use the time derivative $\dot{S}=d S / d t$ with time $t$ for a constant population size of $N=S+I+R$ individuals. The solution of $R(t)$ is given by $R(t)=N-I(t)-S(t)$ which can be calculated using the solution of the ODEs. The susceptible individuals become infected with infection rate $\beta$, recover from the infection with recovery rate $\gamma$ and become susceptible again after waning immunity rate $\alpha$.

In Fig. 1 we show the dynamical behavior of the susceptible, infected and recovered individuals in a given population $N$, when solving the above ODE system.

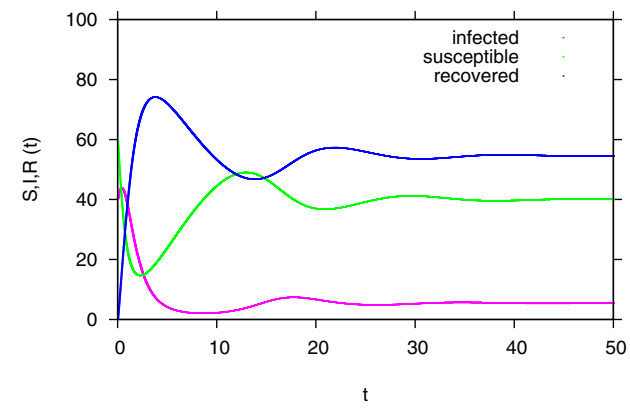

Fig. 1. Time dependent solution simulation for the SIR epidemic model. With a population $N=100$, and starting values $I=40, S=60$ and $R=0$, we fixed $\beta=2.5, \alpha=0.1$, and $\gamma=1$. In green the dynamics for the susceptibles $S(t)$, in pink the dynamics for the infected $I(t)$ and in blue the dynamics of the recovered $R(t)$. Note that $N=100$ allows for the interpretation for the class abundances in percentages.

The basic SIR model has only fixed points as possible stationary solutions, that can be calculated setting the rates of change $\dot{S}$ and $\dot{I}$ to zero. For the disease free equilibrium state, the solution is given by

$$
\begin{aligned}
& I_{1}^{*}=0 \\
& S_{1}^{*}=N
\end{aligned}
$$


and for the disease endemic equilibrium state, the solution is

$$
\begin{aligned}
& I_{2}^{*}=N\left(1-\frac{\gamma}{\beta}\right) \frac{\alpha}{(\alpha+\gamma)} \\
& S_{2}^{*}=N \frac{\gamma}{\beta} .
\end{aligned}
$$

The epidemic dynamic as a function of the parameter $\beta$ shows the spread of the epidemic when $\beta>\gamma$ (see Fig. 2 a)), and its extinction when $\beta<\gamma$ (see Fig. 2 b)).

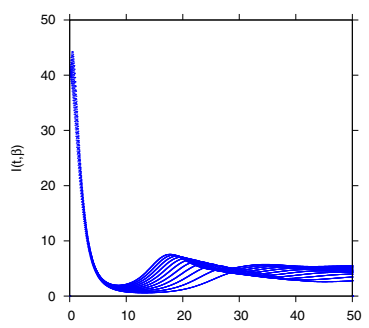

a)

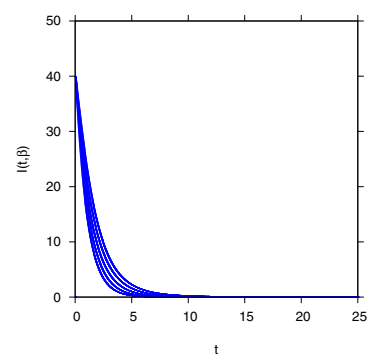

b)

Fig. 2. Epidemic dynamics as a function of $\beta$. With the same initial values as used in Fig. 1, we plot time dependent solutions $I(t)$ for several $\beta$ values. In a) $\beta \in[1.5,2.5]$, with a resolution $\Delta \beta=0.1$ and in b) $\beta \in[0,0.9]$ where $\Delta \beta=0.2$.

In order to analyze the stability of the equilibrium states, we look at the Jacobian matrix and its eigenvalues. Let the dynamics for the state $\underline{x}:=(S, I)$ be $f(\underline{x})$, hence $\frac{d}{d t} \underline{x}=f(\underline{x})$ which explicitly gives $\Delta \underline{x}:=\underline{x}(t)-\underline{x}^{*}$ as a small perturbation around the fixed point $\underline{x}^{*}$. We linearize the dynamic $\frac{d}{d t} \Delta \underline{x}=\frac{\bar{d}}{d t}\left(\underline{x}(t)-x^{*}\right)$ applying Taylor's expansion

$$
\underline{f}\left(\underline{x}^{*}+\Delta \underline{x}\right)=\underline{f}\left(\underline{x}^{*}\right)+\left.\frac{d \underline{f}}{d \underline{x}}\right|_{x^{*}} \cdot(\Delta \underline{x})+\mathcal{O}\left((\Delta x)^{2}\right)
$$

with $f\left(\underline{x}^{*}\right)=0$ for the fixed point and neglecting higher order terms. For our system we have the following linear differential equation system

$$
\left.\frac{d}{d t}\left(\begin{array}{c}
S(t)-S^{*} \\
I(t)-I^{*}
\end{array}\right)=\left(\begin{array}{ll}
\frac{\partial f}{\partial S} & \frac{\partial f}{\partial I} \\
\frac{\partial g}{\partial S} & \frac{\partial g}{\partial I}
\end{array}\right) \mid \begin{array}{l}
S \\
I
\end{array}\right)=\left(\begin{array}{c}
S^{*} \\
I^{*}
\end{array}\right) \cdot\left(\begin{array}{c}
S-S^{*} \\
I-I^{*}
\end{array}\right)
$$

where $f:=(f, g)$ and the Jacobian matrix is explicitly given by

$$
\left(\begin{array}{cc}
-\frac{\beta}{N} I^{*}-\alpha & -\frac{\beta}{N} S^{*}-\alpha \\
\frac{\beta}{N} I^{*} & \frac{\beta}{N} S^{*}-\gamma
\end{array}\right)=: A
$$

where we have to insert for $S^{*}$ and $I^{*}$, the respective steady states. In order to decoupled the linear differential equation system, we diagonalize the matrix $A,(9)$, with the eigenvalue decomposition $A \underline{u}=\lambda \underline{u}, \underline{u}$ is an eigenvector of $A$, and $\lambda$ is an eigenvalue of $A$ corresponding to the eigenvector $\underline{u}$.

The eigenvalues can be calculated setting the determinant of $[A-\lambda \mathbb{1}]$ equal zero. 


\subsection{The disease free equilibrium state}

For the disease free equilibrium state ( $I_{1}^{*}$ and $\left.S_{1}^{*}\right)$, Eq.(3) and Eq. (4), the eigenvalues are given by

$$
\begin{aligned}
& \lambda_{1}=\beta-\gamma \\
& \lambda_{2}=-\alpha .
\end{aligned}
$$

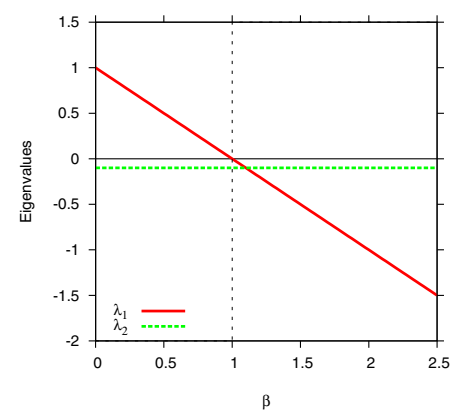

Fig. 3. Eigenvalues for the disease free equilibrium state as functions of $\beta$ when fixing $\alpha=0.1$ and $\gamma=1.0$.

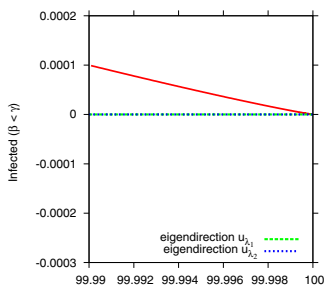

a)

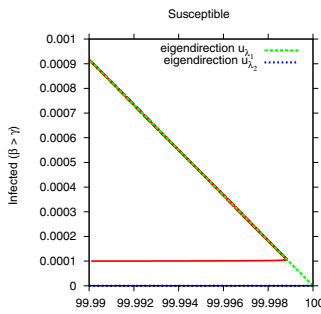

d)

Susceptible

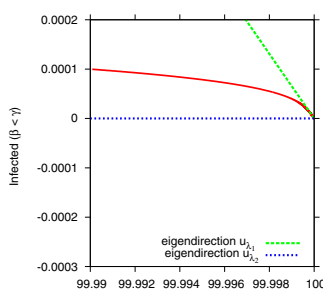

b)

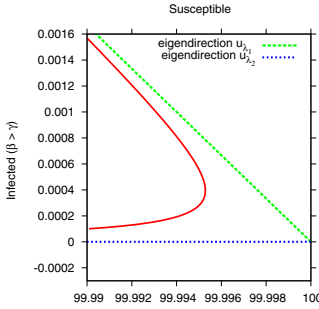

e)

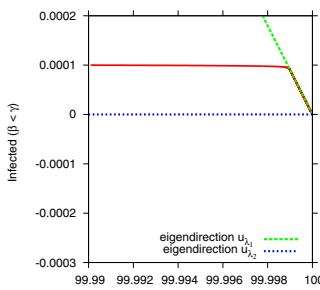

c)

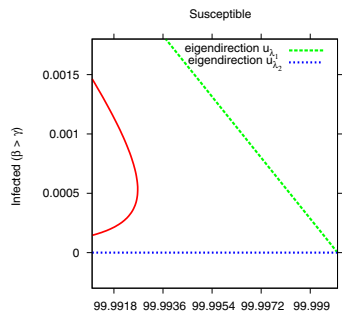

f) Susceptible

Fig. 4. eigenvectors for the disease free equilibrium state in function of $\beta$. For a population $N=100$, where $I=0.0001, S=99.99$, and $R=N-I-S$, in a) $\beta=0.9$, in b) $\beta=0.97$, in c) $\beta=0.999$, in d) $\beta=1.001$, in e) $\beta=1.1$, and in f) $\beta=1.3$.

When looking at Eq. (10) and Eq. (11) we see that for $\beta<\gamma$ both eigenvalues are negative, i.e. the fixed point $I_{1}^{*}$ is stable. The eigenvalues $\lambda_{1}$ and $\lambda_{2}$ are equal at the point $\beta=\gamma-\alpha$ and for $\beta>\gamma, \lambda_{1}$ is positive and $\lambda_{2}$ is negative, therefore the fixed point $I_{1}^{*}=0$ is unstable. The stability of the system changes when one of the eigenvalues of the system becomes zero. At 
this point, $\beta_{c}=\gamma$, when $I_{1}^{*}$ becomes unstable and $I_{2}^{*}$ stable. Fig. 3 shows the eigenvalues for the disease free equilibrium state as functions of $\beta$.

To calculate the corresponding eigenvectors we use $(A-\lambda \mathbb{1}) \underline{u}=0$, with $\lambda_{i}$ and $\underline{u}_{i}=:\left(\begin{array}{l}u_{1 i} \\ u_{2 i}\end{array}\right)$. For the first eigenvalue, $\lambda_{1}$, the correspondent eigenvector $\underline{u}_{1}$ is giving by

$$
\underline{u}_{1}=\frac{1}{\sqrt{1+\left(\frac{\gamma-\beta-\alpha}{\beta+\alpha}\right)^{2}}} \cdot\left(\begin{array}{c}
1 \\
\left(\frac{\gamma-\beta-\alpha}{\beta+\alpha}\right)
\end{array}\right),
$$

and for $\lambda_{2}$, the correspondent eigenvector $\underline{u}_{2}$ is is giving by

$$
\underline{u}_{2}=\left(\begin{array}{l}
1 \\
0
\end{array}\right) \text {. }
$$

In Fig. 4 we show the eigenvectors for the disease free equilibrium state as functions of $\beta$, when fixing $\alpha=0.1$, and $\gamma=1$. We plot the eigenvectors, $\underline{u}_{1}$ (blue line) and $\underline{u}_{2}$ (green line) on top of the trajectory of the infected individuals (red line). Note that $\lambda_{1}=\lambda_{2}$ at $\beta=(\gamma-\alpha)$, i.e. the eigenvectors $\underline{u}_{1}=\underline{u}_{2}$ (see Fig. 4a)). By increasing $\beta$ toward the critical value $\beta_{c}=\gamma$ the trajectory needs longer time to hit the fixed point (see Fig. $4 b$ ) and $4 c$ )). For $\beta>\gamma$, the trajectory goes toward the other fixed point $I_{2}^{*}$ (see Fig. 4d) and 4e)).

\subsection{The disease endemic equilibrium state}

For the disease endemic equilibrium state $\left(I_{2}^{*}\right.$ and $\left.S_{2}^{*}\right)$, Eq. (5) and Eq. (6), the eigenvalues are giving by

$$
\begin{aligned}
& \lambda_{1}=-\frac{\alpha}{2}\left(1+\frac{\beta-\gamma}{\alpha+\gamma}\right)+\sqrt{\left[\frac{\alpha}{2}\left(1+\frac{\beta-\gamma}{\alpha+\gamma}\right)\right]^{2}-(\beta-\gamma) \alpha} \\
& \lambda_{2}=-\frac{\alpha}{2}\left(1+\frac{\beta-\gamma}{\alpha+\gamma}\right)-\sqrt{\left[\frac{\alpha}{2}\left(1+\frac{\beta-\gamma}{\alpha+\gamma}\right)\right]^{2}-(\beta-\gamma) \alpha} .
\end{aligned}
$$

In order to simplify the notation, let $-\frac{\alpha}{2}\left(1+\frac{\beta-\gamma}{\alpha+\gamma}\right)=: a$ and $\left[\frac{\alpha}{2}\left(1+\frac{\beta-\gamma}{\alpha+\gamma}\right)\right]^{2}-(\beta-\gamma) \alpha=: b$. If $b>0$ the eigenvalues are real numbers, giving the contraction or expansion of the trajectories near to the considered fixed point, and can be written as

$$
\begin{aligned}
& \lambda_{1}=a+\sqrt{b} \\
& \lambda_{2}=a-\sqrt{b} .
\end{aligned}
$$

If $b<0$, the eigenvalues

$$
\begin{aligned}
& \lambda_{1}=a+i \sqrt{|b|} \\
& \lambda_{2}=a-i \sqrt{|b|}
\end{aligned}
$$


become complex, where the real part $a$ gives the contraction or expansion, and the imaginary part $i \sqrt{|b|}$ gives the frequency of oscillations of the trajectories spiraling into the fixed point as is shown in Fig. 5. The parabola curve shows the contraction and expansion of the eigenvalues. For $\beta<\gamma$ the fixed $I_{2}^{*}$ point is unstable, with one positive eigenvalue.

For $\beta>\gamma$, the fixed point $I_{2}^{*}$ becomes stable with both eigenvalues negative. The system changes stability when $\beta_{c}=\gamma$ and becomes complex when $\lambda_{1}=\lambda_{2}$ (see Fig. 5).

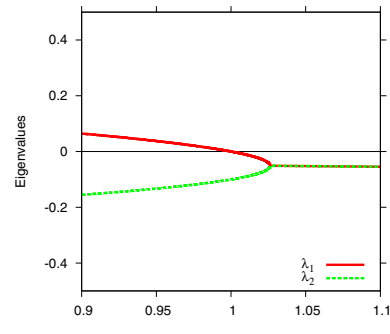

a)

Fig. 5. Plot of the eigenvalues as functions of $\beta$ when fixing $\alpha=0.1$ and $\gamma=1.0$. In a) the real part $a$ of the eigenvalues gives the contraction and expansion of the trajectories. For $\beta<\gamma$ the fixed point is unstable $\left(\lambda_{1}>0\right)$. The system changes stability when $\beta_{c}=\gamma$ and becomes complex when $\lambda_{1}=\lambda_{2}$. Here, the straight green line represents only the real parts of the complex eigenvalues obtained putting $\sqrt{-b}=0$. In $b$ ) the imaginary part of the eigenvalues gives the frequency of the oscillatory behavior on the trajectory toward at the fixed point.

The correspondent eigenvectors of the disease endemic equilibrium state can be calculated for the eigenvalues in the same manner as it was shown above. For the first eigenvalue $\lambda_{1}$, the correspondent eigenvector $\underline{u}_{1}$ is given by

$$
\underline{u}_{1}=\frac{1}{\sqrt{1+\left(\frac{a-\sqrt{b}}{\gamma+\alpha}\right)^{2}}} \cdot\left(\frac{a-\sqrt{b}}{\gamma+\alpha} \cdot \frac{1}{\sqrt{1+\left(\frac{a-\sqrt{b}}{\gamma+\alpha}\right)^{2}}}\right) .
$$

and for the second eigenvalue $\lambda_{2}$, the correspondent eigenvector $\underline{u}_{2}$ is given by

$$
\underline{u}_{2}=\frac{1}{\sqrt{1+\left(\frac{a+\sqrt{b}}{\gamma+\alpha}\right)}} \cdot\left(\begin{array}{c}
1 \\
\frac{a+\sqrt{b}}{\gamma+\alpha}
\end{array}\right) .
$$

In Fig. 6 we show the eigenvectors for the disease endemic equilibrium state in function of $\beta$. For the real eigenvalue the general solution of the linearized system is given by

$$
\underline{x}(t)=C_{1} e^{\lambda_{1} t} \underline{u_{1}}+C_{2} e^{\lambda_{2} t} \underline{u_{2}}
$$

when $\lambda_{1} \neq \lambda_{2}$. By including the respective eigenvalues, Eq. (10) and Eq.(11), we get as a solution

$$
\underline{x}(t)=C_{1} e^{(\beta-\gamma) t} \underline{u_{1}}+C_{2} e^{-\alpha t} \underline{u_{2}}
$$




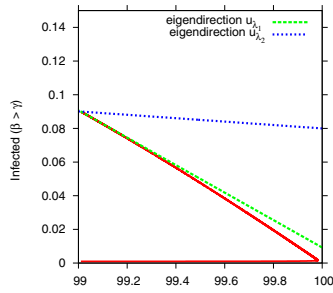

a)

Susceptible

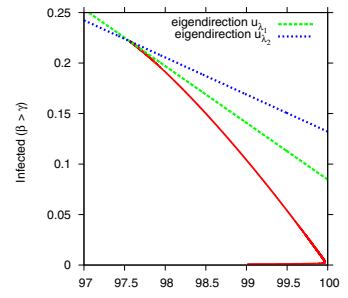

b)

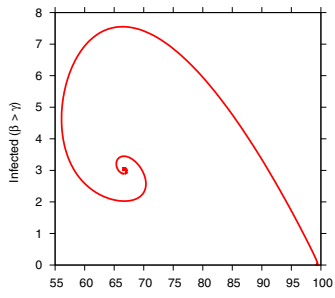

c)

Susceptible

Fig. 6. eigenvectors for the disease endemic equilibrium state in function of $\beta$. For a population $N=100$, where $I=0.001$ and $S=99$, we fixed $\alpha=0.1, \gamma=1$ and vary $\beta$. We plot the eigenvectors, $\underline{u}_{1}$ (blue line) and $\underline{u}_{2}$ (greenline), on top of the trajectory of the infected individuals toward the second fixed point $I_{2}^{*}$. In a) $\beta=1.01$, and in b) $\beta=1.025$. In c) we show the oscillatory trajectory toward the fixed point when $\beta=2.5$

where the eigenvector $u_{1}$ is the driving force for $t \rightarrow \infty$, since $\lambda_{1}>\lambda_{2}$ (see Fig. $4 \mathrm{~b}$ ) to $4 \mathrm{f}$ )). When $\lambda_{1}<\lambda_{2}$ the eigenvector $\underline{u_{2}}$ is the driving force for $t \rightarrow \infty$.

For the special case where $\lambda_{1}=\lambda_{2}=: \lambda$ and therefore the eigenvectors $\underline{u}_{1}=\underline{u}_{2}=: \underline{u}$ the general solution is then given by

$$
\underline{x}(t)=C_{1} e^{\lambda t} \underline{u}+C_{2}\left(t e^{\lambda t} \underline{u}+e^{\lambda t} \underline{w}\right)
$$

where $\underline{w}$ is the so called generalized eigenvector, satisfying $(A-\lambda \mathbb{1}) \underline{w}=\underline{u}$. In this case, for $t \rightarrow \infty$, the eigenvector $\underline{u}$ is again the driving force (see Fig. 4a)).

For the complex eigenvalues, where the real part $a$ gives the contraction or expansion, and the imaginary part $i \sqrt{|b|}$ gives the frequency of oscillations of the trajectories spiraling into the fixed point, the general solution of the linearized system is given by

$$
\underline{x}(t)=2 e^{a t}\left(\left[C_{1} \cos (\sqrt{b} t)-C_{2} \sin (\sqrt{b} t)\right] \underline{u_{1}}-\left[C_{1} \sin (\sqrt{b} t)+C_{2} \cos (\sqrt{b} t)\right] \underline{u_{2}}\right) ，
$$

where $C_{1}$ and $C_{2}$ depend on the initial conditions and $u_{i}$, respectively the real and imaginary parts of the complex eigenvector. This expression shows that the stability of the fixed point depends on the sign of $a$. For detailed information on the solution of a linear two dimensional ODE system, see (Mattheij \& Molenaar, 1996).

The stochastic SIR epidemic is modeled as a time-continuous Markov process to capture population noise. The dynamics of the probability of integer infected and integer susceptibles, while the recovered follow from this due to constant population size, can be give as a master equation (van Kampen, 1992) in the following form

$$
\begin{aligned}
\frac{d p(S, I, t)}{d t} & =\frac{\beta}{N}(S+1)(I-1) \quad p(S+1, I-1, t)+\gamma(I+1) \quad p(S, I+1, t) \\
& +\alpha(N-(S-1)-I) \quad p(S-1, I, t)-\left(\frac{\beta}{N}+\gamma I+\alpha(N-S-I)\right) \quad p(S, I, t)
\end{aligned}
$$

This process can be simulated by the Gillespie algorithm giving stochastic realizations of infected and susceptibles in time (Gillespie, 1976, 1978). 


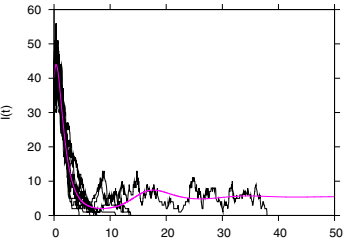

a)

b)

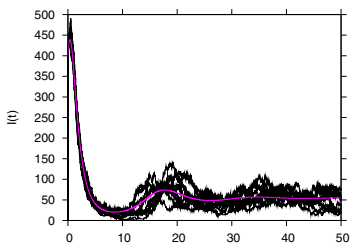

Fig. 7. Stochastic simulations for the basic SIR epidemic model. Here 10 realizations are plotted. We fixed $\alpha=0.1, \gamma=1$ and $\beta=2.5$. The deterministic trajectory is shown (pink line) top of the stochastic realizations for different population size $N$ a. In a) $N=100$, in b) $N=1000$ and in c) $N=100000$.

For mean values of infected $\langle I\rangle$ and susceptibles $\langle S\rangle$, defined as e.g.

$$
\langle I\rangle(t):=\sum_{S=0}^{N} \sum_{I=0}^{N} I p(S, I, t) .
$$

one can calculate the dynamics by inserting the master equation into the definition of the mean values obtaining

$$
\begin{aligned}
\frac{d}{d t}\langle S\rangle & =\alpha\langle R\rangle-\frac{\beta}{N}\langle S I\rangle \\
\frac{d}{d t}\langle I\rangle & =\frac{\beta}{N}\langle S I\rangle-\gamma\langle I\rangle
\end{aligned}
$$

with $\langle R\rangle=N-\langle S\rangle-\langle I\rangle$. For more details on the calculations see e.g. (Stollenwerk \& Jansen, 2010). These equations for the mean dynamics include now due to the nonlinear transition rates in the master equation also higher moments $\langle S \cdot I\rangle$. The simplest approximation to obtain a closed ODE system is to neglect cross-correlations $\langle S \cdot I\rangle-\langle S\rangle \cdot\langle I\rangle \approx 0$, the so-called mean field approximation (originally introduced for spatially extended systems in statistical physics (Stollenwerk et al., 2010)). Hence, the equation system (23) gives with identifying the higher moment $\langle S \cdot I\rangle=\langle S\rangle \cdot\langle I\rangle$ by a product of simple moments gives again the ODE system for SIR system, as it was just presented above. For certain parameter regions the mean field approximation describes the system well in terms of its mean dynamics and only small fluctuations around it. Then the previously shown analysis of the system is appropriate. However, noise can stabilize transients, a feature which becomes important in parameter regions where in the deterministic description a fixed point is reached via decreasing oscillations, as we have observed them in the SIR system. The noisy system would show here continued oscillations (Alonso et al., 2006).

In Fig. 7 we compare the deterministic and stochastic dynamics and we see that the magnitude of stochastic fluctuations decreases when the population size increases. However, the good approximation (see Fig. 7c)) is only achieved when the population size is large enough (see Fig. 7a) where most simulations die out very quickly for small population size).

Almost all mathematical models of diseases start from the same basic premise: that the population can be subdivided into a set of distinct classes. The most commonly used 
framework for epidemiological systems, is still the SIR type model, a good and simple model for many infectious diseases. However, different extensions of the classical single-strain SIR model show a rich dynamic behavior, e.g. (Stone et al., 2007) in measles, or in generalized multi-strain SIR type models to describe the epidemiology of dengue fever (Aguiar et al., 2008).

\section{Dengue fever epidemiology}

Dengue is a viral mosquito-borne infection which in recent years has become a major international public health concern. According to the estimates given by (PDVI, 2011), 3.6 billion ( $55 \%$ of world population) are at risk of acquiring dengue infection (see Fig. 8a)). It is estimated that every year, there are $70-500$ million dengue infections, 36 million cases of dengue fever (DF) and 2.1 million cases of dengue hemorragic fever (DHF), with more than 20.000 deaths per year (CDC, 2011; PDVI, 2011; WHO, 2009). In many countries in Asia and South America DF and DHF has become a substantial public health concern leading to serious social-economic costs.

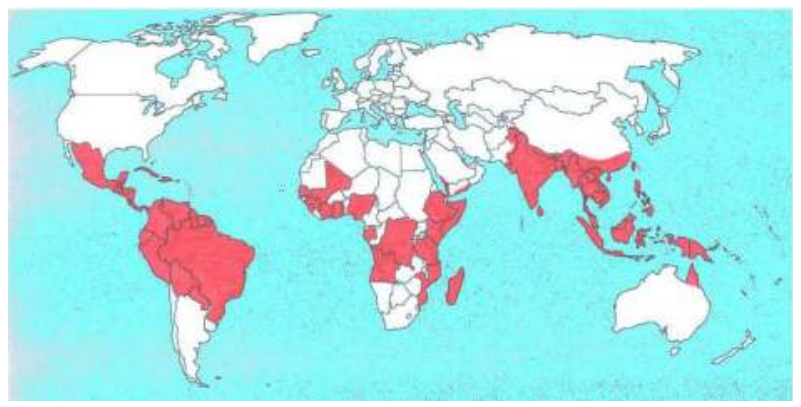

Fig. 8. Worldwide Dengue distribution 2010. In red Countries and areas where dengue has been reported Data source: World Health Organization (WHO) \& Centers for Disease Control and Prevention (CDC). Adapted from (Gubler, 2002; Mackenzie et al., 2004).

Dengue fever is transmitted by the female domestic mosquito Aedes aegypti, although Ae. albopictus and Ae. polynesiensis can also act as transmission vector (Favier et al., 2005). Virus transmission in its simplest form involves the ingestion of viremic blood by mosquitoes and passage to a second susceptible human host. The mosquito becomes infected when taking a blood meal from a viremic person. After an extrinsic incubation period, the mosquito becomes infective and remains so during its entire life span (Rigau-Pérez et al., 1998). As the blood meal stimulates ovoposition, which undergoes at least one, often more, reproductive cycles there is an opportunity of vertical transmission to the eggs, passing the virus to the next generation of mosquitoes (CDC, 2011; Monath, 1994; Rosen et al., 1983).

There are four antigenically distinct dengue viruses, designated DEN-1, DEN-2, DEN-3, and DEN-4 (Guzmán et al., 2010; Halstead, 1994; SES, 2010; WHO, 2009). Infection by one serotype confers life-long immunity to only that serotype and a short temporary cross-immunity period to other serotypes exists. It lasts from three to nine months, when the antibody levels created during the response to that infection would be enough to protect against infection by a different but related serotype (Dejnirattisai et al., 2010; Halstead, 1994; Matheus et al., 2005; SES, 2010; WHO, 2009). Two variants of the disease exist: dengue fever (DF), a non-fatal form 
of illness, and dengue hemorrhagic fever (DHF), which may evolve toward a severe form known as dengue shock syndrome (DSS).

Epidemiological studies support the association of DHF with secondary dengue infection (Guzmán et al., 2000; Halstead, 1982, 2003; Nisalak et al., 2003; Vaughn, 2000), and there is good evidence that sequential infection increases the risk of developing DHF, due to a process described as antibody-dependent enhancement (ADE), where the pre-existing antibodies to previous dengue infection cannot neutralize but rather enhance the new infection.

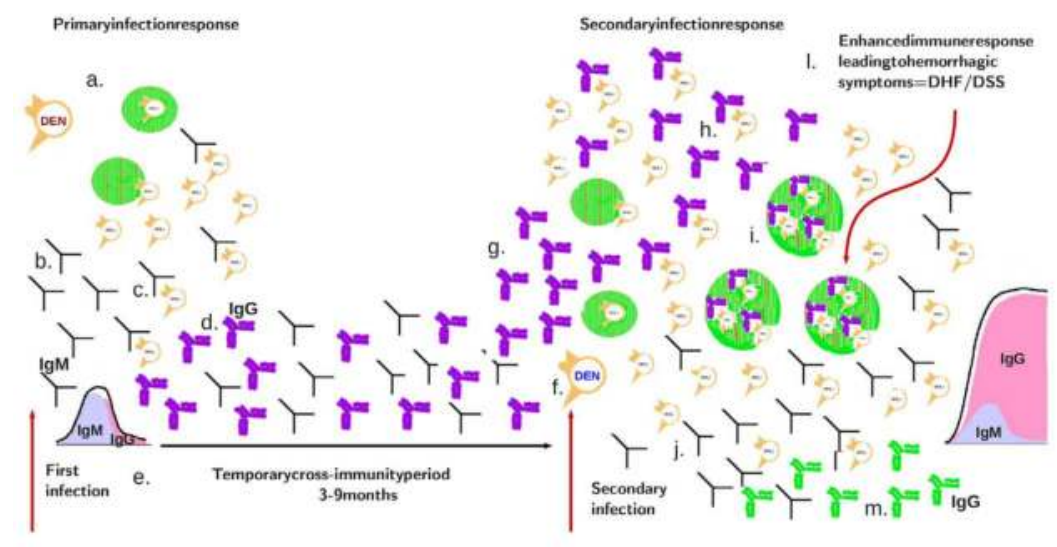

Fig. 9. Scheme of the immunological response on recurrent dengue infections. In (a.) the first infection with a given dengue virus serotype, in (b.) production of antibodies (Immunoglobulin M (IgM)), in (c.) inactivation of the virus and in (d.) production of antibodies (IgG class, the so called memory antibodies). In (e.) the temporary cross immunity period, that lasts between 3-9 months. After that period, the individual can get infected again with another dengue virus serotype (f.). In (g.) the IgG from the previous dengue infection binds to the new virus but do not inactivate them. In (h.) the complex antibody-virus enhances the new infection (i.). In (j.) the production of antibodies (IgM class) which is then able to inactivate the new viruses, leading to (1.), an enhanced immune response, such that hemorrhagic symptoms are observed. In (m.) production of IgG antibodies.

In the first dengue infection virus particles will be captured and processed by so-called antigen presenting cells. These viruses will be presented to T-cells causing them to become activated. And likewise B-cells will encounter their antigen free floating and become activated. B-cells produce antibodies that are used to tag the viruses to encourage their uptake by macrophages and inactivate them. In a secondary infection the antibodies from the first infection will attach to the virus particles but will not inactivate them. The antibody-virus complex suppresses innate immune responses, increasing intracellular infection and generating inflammatory citokines and chemokines that, collectively, result in enhanced disease (Dejnirattisai et al., 2010; Guzmán et al., 2010; Halstead, 1982, 1994, 2003; Mackenzie et al., 2004; WHO, 2009). Fig.9 is an scheme to illustrate the immunological response on recurrent dengue infections.

DF is characterized by headache, retro-orbital pain, myalgia, arthralgia, rash, leukopenia, and mild thrombocytopenia. The symptoms resolve after 27 days. DHF is a potentially 
deadly complication that is characterized by high fever and hemorrhagic phenomenae. DHF develops rapidly, usually over a period of hours, and resolves within 12 days in patients who receive appropriate fluid resuscitation. Otherwise, it can quickly progress to shock (CDC, 2011; WHO, 2009).

Treatment of uncomplicated dengue cases is only supportive, and severe dengue cases requires careful attention to fluid management and proactive treatment of hemorrhagic symptoms (CDC, 2011; WHO, 2009). A vaccine against dengue is not yet available, since it would have to simulate a protective immune response to all four serotypes (Stephenson, 2005), although several candidates of tetravalent vaccines are at various stages of development (WHO, 2011).

Mathematical models describing the transmission of dengue viruses appeared in the literature early as 1970 (Fischer \& Halstead, 1970). More recently, mathematical models describing the transmission of dengue viruses have focused on the ADE effect and temporary cross immunity trying to explain the irregular behavior of dengue epidemics. Such models ultimately aim to be used as a predictive tool with the objective to guide the policies of prevention and control of the dengue virus transmission, including the implementation of vaccination programs when the candidate dengue fever vaccines will be accessible. In the literature the multi-strain interaction leading to deterministic chaos via ADE has been described previously, e.g. (Billings et al., 2007; Ferguson et al., 1999; Schwartz et al, 2005) but neglecting temporary cross immunity. Consideration of temporary cross immunity is rather complicated and up to now not in detail analyzed. Models formulated in (Loureço \& Recker, 2010; Nagao \& Koelle, 2008; Recker et al., 2009; Wearing \& Rohani, 2006), did not investigate closer the possible dynamical structures. In (Aguiar \& Stollenwerk, 2007; Aguiar et al., 2008, 2009,2011 a) by including temporary cross immunity into dengue models with ADE, a rich dynamic structure including deterministic chaos was found in wider and more biologically realistic parameter regions.

\section{Multi-strain models motivated by dengue fever epidemiology: a review}

Multi-strain dynamics are generally modelled with SIR-type models and have demonstrated to show critical fluctuations with power law distributions of disease cases, exemplified in meningitis and dengue epidemiology (Massad et al., 2008; Stollenwerk \& Jansen, 2003; Stollenwerk et al., 2004). Dengue models including multi-strain interactions via ADE but without temporary cross immunity period e.g. (Billings et al., 2007; Ferguson et al., 1999; Schwartz et al, 2005) have shown deterministic chaos when strong infectivity on secondary infection was assumed. The addition of the temporary cross immunity period in such models shows a new chaotic attractor in an unexpected parameter region of reduced infectivity on secondary infection (Aguiar \& Stollenwerk, 2007; Aguiar et al., 2008, 2009, 2011 a), i.e. deterministic chaos was found in a wider parameter regions. This indicates that deterministic chaos is much more important in multi-strain models than previously thought, and opens new ways to data analysis of existing dengue time series, as will be shown below. It offers a promising perspective on parameter values inference from dengue cases notifications.

The basic multi-strain model divides the population into ten classes: susceptible to both strains, 1 and $2(S)$, primarily infected with strain one $\left(I_{1}\right)$ or strain two $\left(I_{2}\right)$, recovered from the first infection with strain one $\left(R_{1}\right)$ or strain two $\left(R_{2}\right)$, susceptible with a previous infection with strain one $\left(S_{1}\right)$ or strain two $\left(S_{2}\right)$, secondarily infected with strain one when the first 
infection was caused by strain two $\left(I_{21}\right)$ or for second time infected with strain two when the first infection was caused by strain one $\left(I_{12}\right)$. Notice that infection by one serotype confers life-long immunity to that serotype. Then the individuals recover from the secondary infection $(R)$.

To capture differences in primary infection by one strain and secondary infection by another strain we consider a basic two-strain SIR-type model for the host population, which is only slightly refined as opposed to previously suggested models for dengue fever (Billings et al., 2007; Ferguson et al., 1999; Schwartz et al, 2005).

The stochastic version of the multi-strain dengue model is now in complete analogy to the previously described SIR model, and the mean field ODE system for the multi-strain dengue model can be read from the following reaction scheme (24), describing the transitions for first infection with strain 1 and secondary infection with strain 2 , and for the reverse process, where the first infection is caused by strain 2 and the secondary infection is caused by strain 1, the same reaction scheme can be used to describe the transitions by just changing labels.

$$
\begin{aligned}
& S+I_{1} \stackrel{\beta}{\longrightarrow} I_{1}+I_{1} \\
& S+I_{21} \stackrel{\phi \beta}{\longrightarrow} I_{1}+I_{21} \\
& I_{1} \stackrel{\gamma}{\longrightarrow} R_{1} \\
& R_{1} \stackrel{\alpha}{\longrightarrow} S_{1} \\
& S_{1}+I_{2} \stackrel{\beta}{\longrightarrow} I_{12}+I_{2} \\
& S_{1}+I_{12} \stackrel{\phi \beta}{\longrightarrow} I_{12}+I_{12} \\
& I_{12} \stackrel{\gamma}{\longrightarrow} R
\end{aligned}
$$

The demographic transitions are $S, I_{1}, I_{2}, R_{1}, R_{2}, S_{1}, S_{2}, I_{12}, I_{21}, R \stackrel{\mu}{\longrightarrow} S$ defining the system of two strains completely (for more information on the deterministic ODE system and its parametrization, see (Aguiar et al., 2011 a)).

The complete system of ordinary differential equations for the two strain epidemiological system is given by Eq. system (25) and the dynamics are described as follows. Susceptibles to both strains can get the first infection with strain one or strain two with force of infection $\frac{\beta I}{N}$ when the infection is acquired via an individual in his first infection or $\frac{\phi \beta I}{N}$ when the infection is acquired via an individual in his second infection. They recover from the first infection with a recovery rate $\gamma$, conferring full and life-long immunity against the strain that they were exposed to, and also a short period of temporary cross-immunity $\alpha$ against the other strain, becoming susceptible to a second infection with a different strain. The susceptible with a previous infection gets the secondary infection with force of infection $\frac{\beta I}{N}$ or $\frac{\phi \beta I}{N}$ depending on whom (individual on his primary or secondary infection) is transmitting the infection. Then, with recovery rate $\gamma$, the individuals recover and become immune against all strains. We assume no epidemiological asymmetry between strains, i.e. infections with strain one or strain two contribute in the same way to the force of infection. Here, the only relevant difference concerning disease transmissibility is that the force of infection varies accordingly to the number of previous infections the hosts have experienced. The parameter $\phi$ in our model, is the ratio of secondary infection contribution to the force of infection. For more 
information on the parametrization of ADE and secondary dengue infection by $\phi$, see (Aguiar et al., 2008; Ferguson et al., 1999). The parameter values are given in Table 1, if not otherwise explicitly stated.

$$
\begin{aligned}
\dot{S} & =-\frac{\beta}{N} S\left(I_{1}+\phi I_{21}\right)-\frac{\beta}{N} S\left(I_{2}+\phi I_{12}\right)+\mu(N-S) \\
\dot{I_{1}} & =\frac{\beta}{N} S\left(I_{1}+\phi I_{21}\right)-(\gamma+\mu) I_{1} \\
\dot{I_{2}} & =\frac{\beta}{N} S\left(I_{2}+\phi I_{12}\right)-(\gamma+\mu) I_{2} \\
\dot{R_{1}} & =\gamma I_{1}-(\alpha+\mu) R_{1} \\
\dot{R_{2}} & =\gamma I_{2}-(\alpha+\mu) R_{2} \\
\dot{S_{1}} & =-\frac{\beta}{N} S_{1}\left(I_{2}+\phi I_{12}\right)+\alpha R_{1}-\mu S_{1} \\
\dot{S_{2}} & =-\frac{\beta}{N} S_{2}\left(I_{1}+\phi I_{21}\right)+\alpha R_{2}-\mu S_{2} \\
\dot{R} & =\gamma\left(I_{12}+I_{21}\right)-\mu R, \\
\dot{I_{21}} & =\frac{\beta}{N} S_{1}\left(I_{2}+\phi I_{12}\right)-(\gamma+\mu) I_{12} \\
\dot{R} &
\end{aligned}
$$

\begin{tabular}{llll}
\hline Par. Description & Values & Ref \\
\hline$N$ & population size & 100 & - \\
$\mu$ & new born susceptible rate & $1 / 65 y$ & (UNWPP, 2008) \\
$\gamma$ & recovery rate & $52 y^{-1}$ & (Gubler et al., 1981; WHO, 2009) \\
$\beta$ & infection rate & $2 \gamma$ & (Ferguson et al., 1999) \\
$\alpha$ & temporary cross-immunity rate & $2 y^{-1}$ & (Matheus et al., 2005; SES, 2010) \\
$\phi$ & ratio of contrib. to force of inf. & variable & -
\end{tabular}

Table 1. Parameter set, rates given in units per year, ratio without unit

The stationary states can be calculated analytically by setting the time derivatives in Eq. system (25) to zero, 


$$
\begin{aligned}
& S^{*}=\frac{\mu N-(\gamma+\mu)\left(I_{1}^{*}+I_{2}^{*}\right)}{\mu} \\
& I_{21}^{*}=\frac{1}{\phi_{1}}\left(\frac{N}{\beta_{1} S^{*}}(\gamma+\mu)-1\right) I_{1}^{*} \\
& I_{12}^{*}=\frac{1}{\phi_{2}}\left(\frac{N}{\beta_{2} S^{*}}(\gamma+\mu)-1\right) I_{2}^{*} \\
& S_{1}^{*}=\frac{(\gamma+\mu) I_{12}^{*}}{\left(I_{2}^{*}+\phi_{2} I_{12}^{*}\right)} \frac{N}{\beta_{2}} \\
& S_{2}^{*}=\frac{(\gamma+\mu) I_{21}^{*}}{\left(I_{1}^{*}+\phi_{1} I_{21}^{*}\right)} \frac{N}{\beta_{1}} \\
& R_{1}^{*}=\frac{\gamma}{\alpha+\mu} I_{1}^{*} \\
& R_{2}^{*}=\frac{\gamma}{\alpha+\mu} I_{2}^{*},
\end{aligned}
$$

where still the stationary values of $I_{1}^{*}$ and $I_{2}^{*}$ have to be determined.

The solution of coexistence of both strains for $I_{1}=I_{2}=I^{*}$ is given by the following expression

$$
\begin{aligned}
I_{1}^{*}=I_{2}^{*} & =-\left[\frac{\frac{\alpha \gamma}{(\alpha+\mu)(\gamma+\mu)} \phi+\left(\frac{(\gamma+\mu)}{\beta}-3\right)}{4 \frac{(\gamma+\mu)}{\mu}\left(1-\frac{\alpha \gamma}{(\alpha+\mu)(\gamma+\mu)} \phi\right)}\right] N \\
& -\sqrt{\left.\frac{N^{2}\left[\frac{\alpha \gamma}{4}\left[\frac{(\alpha+\mu)(\gamma+\mu)}{2 \frac{(\gamma+\mu)}{\mu}\left(1-\frac{\alpha \gamma}{\beta}(\alpha+\mu)(\gamma+\mu)\right.} \phi\right)\right.}{(\gamma+\mu)}\right]^{2}\left[\frac{N^{2} \mu\left(\frac{(\gamma+\mu)}{\beta}-1\right)}{2 \frac{(\gamma+\mu)^{2}}{\mu}\left(1-\frac{\alpha \gamma}{(\alpha+\mu)(\gamma+\mu)} \phi\right)}\right]},
\end{aligned}
$$

and the solution of the extinction of one of the strains is as follows

$$
\begin{aligned}
& I_{1}^{*}=\frac{\mu N(\beta-(\gamma+\mu))}{(\gamma+\mu) \beta} \\
& I_{2}^{*}=0 .
\end{aligned}
$$

Finally, the stationary value of $R^{*}$, when hosts have been recovered from both strains, is given by the balance equation for the total population size $N$, explicitly

$$
R^{*}=N-\left(S^{*}+I_{1}^{*}+I_{2}^{*}+R_{1}^{*}+R_{2}^{*}+S_{1}^{*}+S_{2}^{*}+I_{12}^{*}+I_{21}^{*}\right) .
$$

The time series for $\phi<1$ shows that the total number of infected $I:=I_{1}+I_{2}+I_{12}+I_{21}$ stays quite away from zero, avoiding the chance of extinction in stochastic systems with reasonable 
system size (see Fig. 10 a)). The parameter region previously considered to model ADE effects on dengue epidemiology, i.e. $\phi>1$, leads to rather low troughs for the total number of infected giving unrealistically low numbers of infected. In Fig. $10 \mathrm{~b}$ ) the logarithm of total number of infected goes as low as -70 for $\phi=2.7$ in the chaotic region of $\phi>1$. Population fluctuations would in this case drive almost surely the system to extinction.

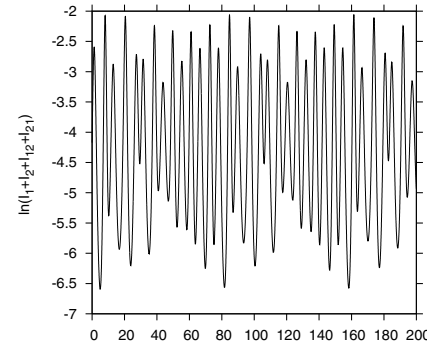

a)

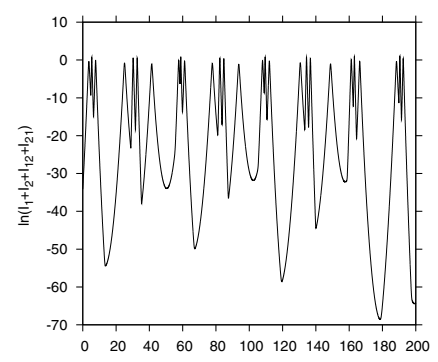

b)

$\mathrm{t}$

Fig. 10. Time series of the logarithm of the overall infected $(\ln (I))$ comparison: a) simulation for $\phi=2.7$ and b) simulation for $\phi=0.6$ for the same time interval.

The state space plots in terms of the variables $S$ and the logarithm of the total number of infected $I$ show a rich dynamical behavior with bifurcations from fixed point to limit cycles, till completely irregular behavior (see Fig. 11).

a)

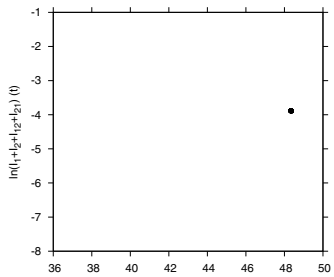

$\mathrm{S}(\mathrm{t})$

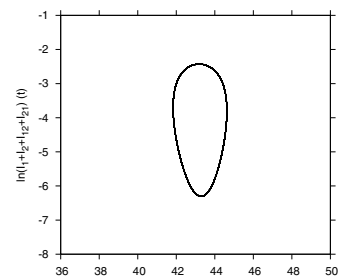

b)

$\mathrm{S}(\mathrm{t})$

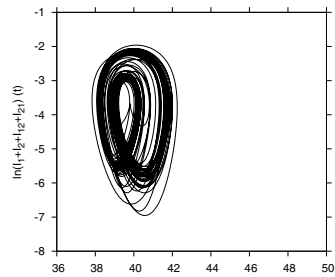

c)

Fig. 11. Attractors for various values of $\phi<1$ : a) fixed point for $\phi=0.1$, and b) limit cycle for $\phi=0.4$, and c) chaotic attractor for $\phi=0.6$.

Looking for higher values of $\phi$, the chaotic attractor becomes unstable, just leaving simple limit cycles as attractors for large parameter regions beyond $\phi=1$ (Aguiar \& Stollenwerk, 2007; Aguiar et al., 2008). Only for much higher values of $\phi>>1$, another chaotic attractor appears, the classical "ADE chaotic attractor" (Aguiar \& Stollenwerk, 2007; Aguiar et al., 2008; Ferguson et al., 1999).

The bifurcation diagram was obtained plotting the local extrema of $\ln (I)$ over the varying parameter $\phi$ (see Fig. 12). Fixed points appear as one dot per parameter value, limit cycles appear as two dots, double-limit cycles as four dots, more complicated limit cycles as more dots, and chaotic attractors as continuously distributed dots for a single $\phi$ value (Ruelle, 1989). We observe two chaotic windows, one for $\phi<1$, where this dynamical behavior has never been described before, and also another one for $\phi>1$ (see Fig. 12a)) where the minimal values go to very low numbers of infected, which already has been described (see Fig. 12b)) in previous publications (Billings et al., 2007; Ferguson et al., 1999; Schwartz et al, 2005). In Fig. $12 \mathrm{~b})$ the dynamical behavior was obtained when neglecting the temporary cross-immunity 


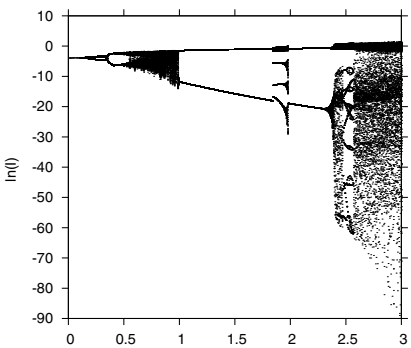

a)

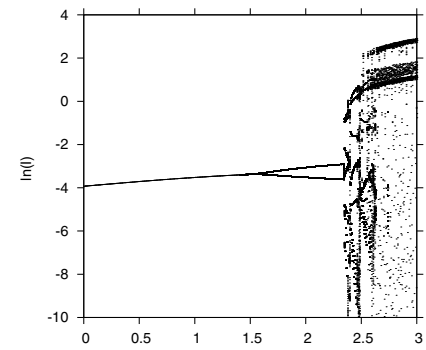

b)

Fig. 12. Bifurcation diagram for the local extrema of the overall infected with changing parameter $\phi$. In a) $\alpha=2$ (six month) and in b) $\alpha=52$ (one week).

period, i.e. by putting $\alpha \rightarrow \infty$. The recovered individuals can be immediately infected with another strain, whereas consideration of temporary cross-immunity brings a new chaotic attractor found first by Aguiar et al. (Aguiar \& Stollenwerk, 2007; Aguiar et al., 2008).

This finding encourages to look closer to the parameter region of $\phi<1$, when dengue patients in a secondary infection evolving to severe disease because of the ADE phenomenon contribute less to the force of infection, and not more, as previous models suggested. This assumption is likely to be more realistic for dengue fever since the possible severity of a secondary infection may hospitalize people, not contributing to the force of infections as much as people with first infection.

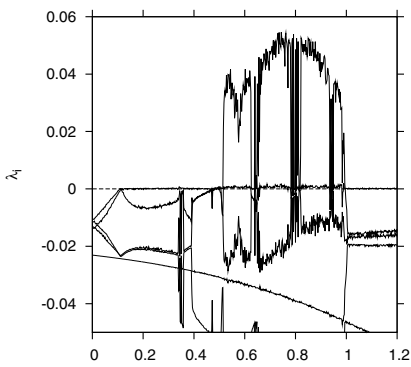

a)

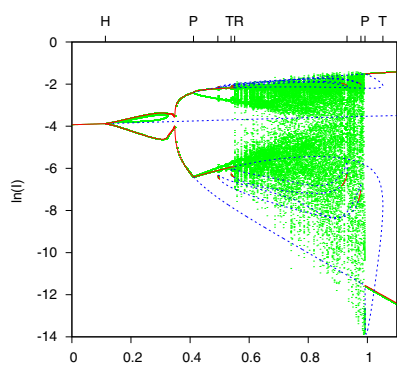

b)

Fig. 13. In a) spectrum of the four largest Lyapunov exponents with changing parameter $\phi$ and fixed $\alpha=2$. In $\mathrm{b}$ ) we show the one-parameter bifurcation diagram with temporary cross-immunity rate $\alpha=2$ and varying the ratio of secondary infection contribution to the force of infection $\phi$. Solid lines denote stable equilibria or limit cycles, and dashed lines unstable equilibria or limit cycles.

We quantify the attractor structure, fixed point, limit cycle or chaotic attractor etc., by calculating Lyapunov exponents (Ott, 1993; Ruelle, 1989), which were calculated using an iterated technique along a trajectory using the QR decomposition algorithm via Householder matrices (see (Aguiar et al., 2008; Holzfuss \& Lauterborn, 1989; Holzfuss \& Parlitz, 1991)). Lyapunov exponents are essentially a generalization of eigenvalues determining stability versus instability along trajectories. A negative largest Lyapunov exponent indicates a stable fixed point as attractor, a zero largest Lyapunov exponent indicates a stable limit cycle and a positive largest Lyapunov exponent indicates a chaotic attractor. Fig. 13a) shows the 
largest four Lyapunov exponents as a function of $\phi$. We observe that for small $\phi$ up to 0.1 all four Lyapunov exponents are negative, indicating the stable fixed point solution. Then follows a region up to $\phi=0.5$ where the largest Lyapunov exponent is zero, characteristic for stable limit cycles. Above $\phi=0.5$ a positive Lyapunov exponent, clearly separated from the second largest Lyapunov exponent being zero, indicates deterministically chaotic attractors. In the chaotic window between $\phi=0.5$ and $\phi=1$ also periodic windows appear, giving a zero largest Lyapunov exponent. These findings are in good agreement with the numerical bifurcation diagram, Fig. 13b).

A further analysis of the bifurcation structure, in the region of interest of $\phi<1$, was performed using the numerical software AUTO (AUTO, 2009). Various bifurcations were found: Hopf bifurcation $H(\phi=0.11326)$, pitchfork bifurcations $P(\phi=0.41145,0.99214)$, torus bifurcation $\operatorname{TR}(\phi=0.55069)$ and tangent bifurcations $T(\phi=0.4 .9406,0.53874,0.93103,0.97825,1.05242)$. In addition to this main bifurcation pattern we found two isolas, consisting of isolated limit cycles existing between two tangent bifurcations (see Fig. 13b), for more information on the isolas see (Aguiar et al., 2008, 2009). These results agree very well with the simulation results shown in the bifurcation diagram for the maxima and minima of the overall infected in Fig. $12 a)$.

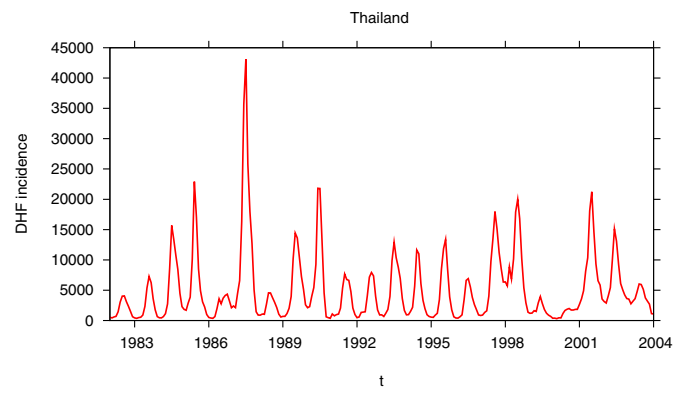

Fig. 14. Time series of DHF incidence in Thailand.

Dengue fever epidemiology is characterized as a yearly cycle of incidences (see Fig. ??), therefore in order to be able to reproduce the yearly cycle in dengue incidence seasonal forcing and a low import of infected have to be included in the models. The first recorded epidemic of DHF in Thailand (population of approximately 66 million people (Wikipedia, 2011)) was in 1958 (WHO, 2009). The co-circulation of all four dengue serotypes and their capacity to produce severe dengue disease was demonstrated as early as 1960 in Bangkok, Thailand (Halstead et al., 1969). DHF occurred first only in Bangkok, but was disseminated to the whole region during the 1970s (Chareonsook et al., 1999; Gubler, 2002; Halstead et al., 1969). Physicians in Thailand are trained to recognize and treat dengue fever and practically all cases of DHF and DSS are hospitalized. A system for reporting communicable diseases including DHF/DSS was considered fully installed in 1974 and the data bank of DHF and DSS is available at the Ministry of Public Health, Bangkok (Chareonsook et al., 1999).

We extend the previously studied non-seasonal model by adding seasonal forcing, mimicking the vectorial dynamics, and a low import of infected individuals, which is realistic in the dynamics of infectious diseases, in order to get a more realistic pattern of dengue fever epidemics, with irregular, yearly and smooth outbreaks. 


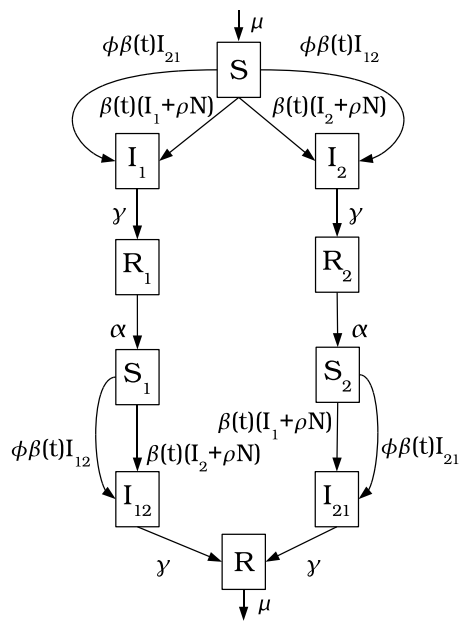

Fig. 15. The state flow diagram for the seasonal multi-strain model. The transition rate $\mu$ coming out of the class $R$ represents the death rates of all classes, $S, I_{1}, I_{2}, R_{1}, R_{2}, S_{1}, S_{2}, I_{12}, I_{21}, R$, getting into the class $S$ as a birth rate.

The seasonal multi-strain model is represented in Fig. 15 by using a state flow diagram where the boxes represent the disease related stages and the arrows indicate the transition rates. In the same manner as described for the non-seasonal, the population is divided into ten classes, with constant size $N=S+I_{1}+I_{2}+R_{1}+R_{2}+S_{1}+S_{2}+I_{12}+I_{21}+R$. The complete system of ordinary differential equations for the seasonal multi-strain epidemiological can be written as shown in system (25), with the difference that now the parameter $\beta$ takes the seasonal forcing into account as a cosine function given explicitly by

$$
\beta(t)=\beta_{0} \cdot(1+\eta \cdot \cos (\omega \cdot t)),
$$

where $\beta_{0}$ is the infection rate, and $\eta$ is the degree of seasonality. In this model, a susceptible individual can become infected also by meeting an infected individual from an external population (hence $(\beta / N \cdot S \cdot I)$ goes to $(\beta / N \cdot S \cdot(I+\rho \cdot N)))$ contributing to the force of infection with an import parameter $\rho$.

The parameters are fixed, temporary cross immunity rate $\alpha=2 y^{-1}$, recovery rate $\gamma=52 y^{-1}$, infection rate $\beta_{0}=2 \cdot \gamma$, seasonality $\eta=0.35$, import factor $\rho=10^{-10}$, birth and death rate $\mu=1 / 65 y$ and the ratio of secondary infection contribution to the force of infection $\phi=0.9$.

In Fig. 16a) the time series simulation results for the total number of infected $\left(I_{1}+I_{2}+I_{12}+\right.$ $I_{21}$ ) in the non-seasonal system, previously studied in (Aguiar et al., 2008), is shown. Besides showing an irregular pattern of outbreaks that happens every 5 years, the non-seasonal system and its time series are not able to represent dengue fever epidemiology that is characterized as a yearly cycle of incidences. By adding low seasonality into the system, the epidemic outbreaks appear every year (see (Aguiar et al., 2011 a)). However, between two large outbreaks there is a very low number of cases in subsequent years, which is also not data alike. In Fig. 7c), the time series simulation in the high seasonal system with a low import of infected contributing to the force of infection is shown. The addition of import into the 
a)

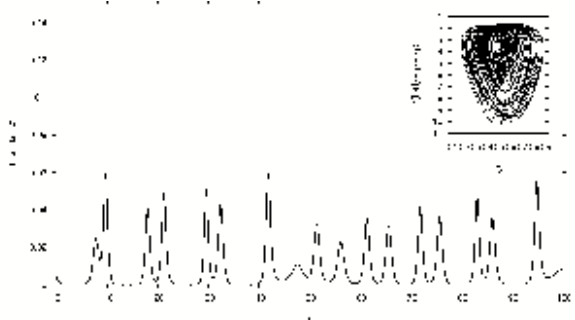

b)

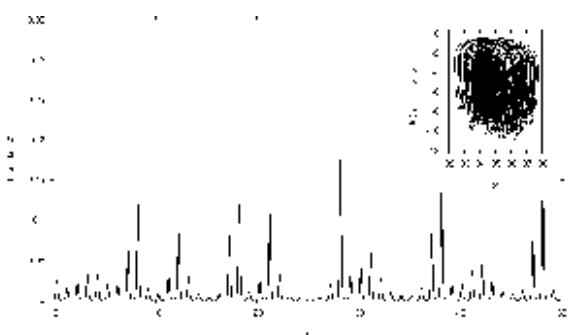

Fig. 16. Time series simulations. In a) time series simulation for the non-seasonal model $(\eta=0)$. In b) time series simulation for the seasonal model with a low import of infected. Here, the degree of seasonality is $\eta=0.35$ and the import of infected $\rho=10^{-10}$.

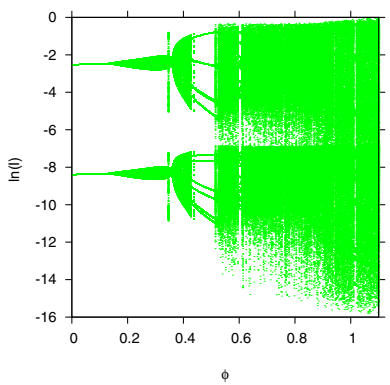

Fig. 17. Bifurcation diagram for the seasonal model with import. Here, the degree of seasonality $\eta=0.35$ and the import factor $\rho=10^{-10}$.

seasonal system gives a much more realistic pattern of dengue fever epidemics, with irregular, yearly and smooth outbreaks. The system has a reasonable size (the number of infected stays quite away from zero), avoiding the chance of extinction in stochastic systems. For detailed analysis on the attractors in state space for the seasonal model, see (Aguiar et al., 2011 a). The bifurcation diagram for the seasonal model with import is shown in Fig. 17.

For the seasonal model with import AUTO predicted a torus bifurcation TR at $\phi=0.13$, and at $\phi=0.522$ which are also predicted very well when comparing with the results given by the Lyapunov exponent calculation. In the limiting case where the amplitude of the seasonal forcing is zero, the torus bifurcation $T R$ of the seasonally forced system coincides with the Hopf bifurcation $H$ of the non-seasonal system, as was shown in (Aguiar et al., 2011 a). 


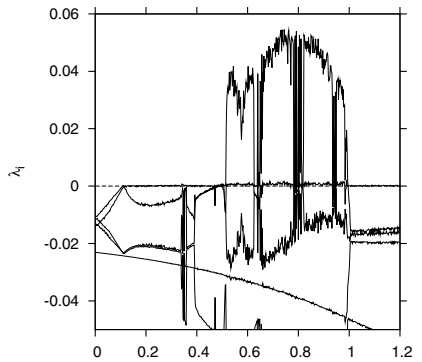

a)

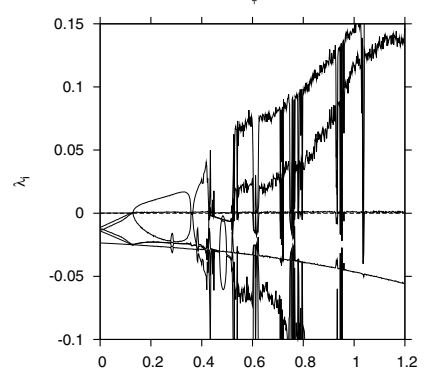

c)

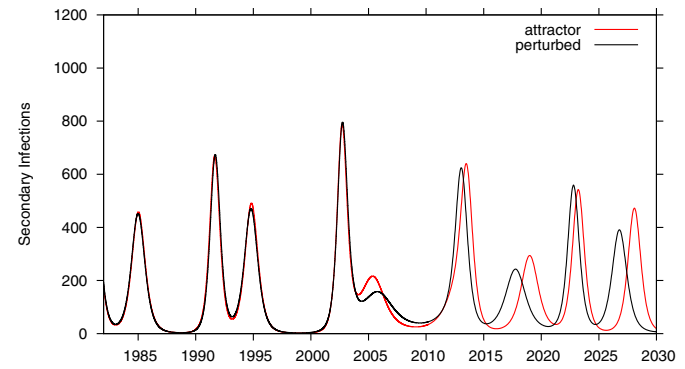

b)

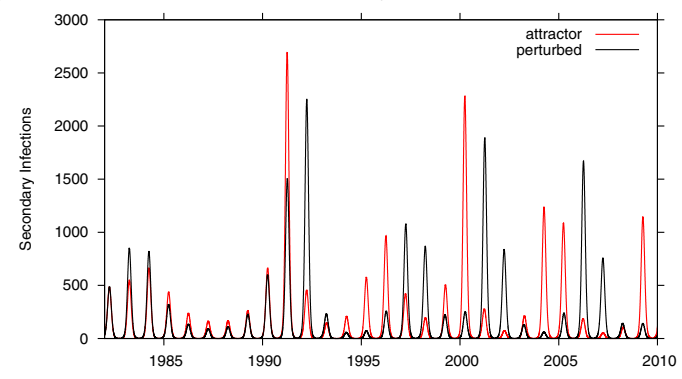

d)

Fig. 18. Qualitative insight into the predictability in the monthly time series. In a) the Lyapunov spectrum and in b) the time series for the non-seasonal model. In c) the Lyapunov spectrum and in d) the time series for the seasonal model with import.

In Fig. 18 the Lyapunov spectrum for both non-seasonal model and the seasonal model with import are shown and compared concerning the prediction horizon of the monthly peaks in the multi-strain dengue model time series. We take as an example the Dominant Lyapunov Exponent (DLE) for $\phi=0.9$ in the region where the system is chaotic (positive DLE). For the non-seasonal system, the DLE $=0.04$ giving around 25 years of prediction horizon in the monthly time series (see Fig. 18b)), whereas for the seasonal system with import,the DLE= 0.118 giving around 8.5 years of prediction horizon in the monthly time series. It is clear that the addition of seasonal forcing into the system by itself decreases the practical predictability, however, the addition of a low import into the seasonally forced system helps to get a more complex dynamics and a better prediction horizon in the monthly time series. In order to get a qualitative insight into the predictability in the monthly sampled time series, i.e. to show how the original system behaves under a small perturbation we plot two different trajectories of the same system (for the non-seasonal model in Fig. 18b), and for the seasonal model with import of infected in Fig. 18d)), where the perturbed system (black line) is compared with the original model simulation (red line). To get the trajectory of the perturbed system, we keep the last point of the transient of the original system and use those values as starting values to compute the new and perturbed trajectory. The perturbation is given by $S_{p}=S+R \cdot \epsilon$ and $R_{p}=R \cdot(1.0-\epsilon)$, where $S_{p}$ is the susceptibles perturbed and $R_{p}$ is the recovered perturbed with $\epsilon=0.001$. (for details on the perturbed system see (Aguiar et al., 2011 a)).

The inspection of the available DHF incidence data in Thailand shows a smooth behavior with a well defined maximum each year of irregular hight for the Northern Provinces. 


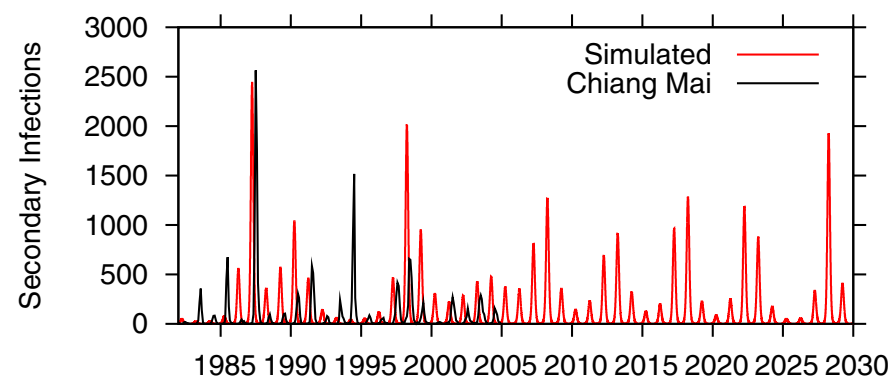

t

Fig. 19. Empirical DHF incidence data matched with the model simulation.

We take the Province of Chiang Mai as a case study where the empirical DHF incidence data and the time series simulation for the seasonal model with import are compared (see Fig. 19)). The seasonal model with import shows complex dynamics and qualitatively a very good result when comparing empirical DHF and simulations. However, the extended model needs to be parametrized on data referring to incidence of severe disease.

\section{Discussions}

In this chapter we presented the properties of the basic SIR epidemic model for infectious diseases with a summary of the analysis of the dynamics, identifying the thresholds and equilibrium points in order to introduce notation, terminology. The results that were generalized to more advanced models motivated by dengue fever epidemiology.

The epidemiology of dengue fever was described presenting the relevant biological features that are taken into the modeling process. Then, multi-strain models previously described in the literature were presented. We focused in a minimal model motivated by dengue fever epidemiology, formulated first by Aguiar et al. (Aguiar \& Stollenwerk, 2007), where the notion of at least two different strains is needed to describe differences between primary infections, often asymptomatic, and secondary infection, associated with the severe form of the disease. We discussed the role of seasonal forcing and the import of infected individuals in such systems, the biological relevance and its implications for the analysis of the available dengue data. The extended model (Aguiar et al., 2011 a) shows complex dynamics and qualitatively a good agreement between empirical DHF monitoring data and the obtained model simulation. This suggests that the used parameter set can be the starting set for a more detailed parameter estimation procedure. Such a technical parameter estimation is notoriously difficult for chaotic time series but temporally local approaches are possible (He et al., 2010; Ionides et al., 2006). At the moment only such minimalistic models have a chance to be qualitatively understood well and eventually tested against existing data.

The introduction of stochasticity is needed to explain the fluctuations observed in some of the available data sets, revealing a scenario where noise and complex deterministic skeleton strongly interact (Aguiar et al., 2011 b). For large enough population size, the stochastic system can be well described by the deterministic skeleton gaining insight into the relevant parameter values purely on topological information of the dynamics, rather than classical 
parameter estimation of which application is in general restricted to fairly simple dynamical scenarios.

\section{Conclusions}

Being able to predict future outbreaks of dengue in the absence of human interventions is a major goal if one wants to understand the effects of control measures. Even after a dengue virus vaccine has become accessible, this holds true for the implementation of a vaccination program. For example, to perform a vaccine trial in a year with normally low numbers of cases would make statistical tests of vaccine efficacy much more difficult than when it was performed in a year with naturally high numbers of cases. Thus predictability of the next season's hight of the dengue peak on the basis of deterministic balance of infected and susceptible would be of major practical use.

\section{Acknowledgments}

The research presented in this book chapter has been supported by the Fundação para a Ciência e a Tecnologia, FCT grant SFRH/BD/43236/2008, and has been further supported by the Portuguese FCT project PTDC/MAT/115168/2009 and by the EU project EPIWORK under Framework Program 7. The work was carried out at Centro de Matemática e Aplicações Fundamentais, Science Faculty, Lisbon University, Portugal.

\section{References}

Aguiar, M. \& Stollenwerk, N. (2007). A new chaotic attractor in a basic multi-strain epidemiological model with temporary cross-immunity. arXiv:0704.3174v1 [nlin.CD].

Aguiar, M., Kooi, B. W. \& Stollenwerk, N. (2008). Epidemiology of Dengue Fever: A Model with Temporary Cross-Immunity and Possible Secondary Infection Shows Bifurcations and Chaotic Behaviour in Wide Parameter Regions. Math. Model. Nat. Phenom., 4, 48-70, ISSN 0973-5348.

Aguiar, M., Stollenwerk, N. \& Kooi, B. W. (2009). Torus bifurcations, isolas and chaotic attractors in a simple dengue model with ADE and temporary cross immunity. International Journal of Computer Mathematics, 86, 1867-1877, ISSN 0020-7160.

Aguiar, M., et al. (2011 a). The role of seasonality and import in a minimalistic multi-strain dengue model capturing differences between primary and secondary infections: complex dynamics and its implications for data analysis. Journal of Theoretical Biology, 289, 181-196, ISSN 0022-5193.

Aguiar, M., Kooi B. W., \& Stollenwerk N. (2011 b). Scaling of stochasticity in DHF epidemics. Under review.

Alonso, D., McKane, A. \& Pascual, M. (2006). Stochastic Amplification in Epidemics. Journal of the Royal Society Interface, 4, 575-582, ISSN 1742-5689.

Anderson, R. M. \& May, R. M. (1979). Population biology of infectious diseases: Part I. Nature, 280, 361-67, ISSN 0028-0836.

Doedel J. E. and Oldeman, B. (2009). AUTO 07P âĂŞ Continuation and bifurcation software for ordinary differential equations. Technical Report: Concordia University, Montreal, Canada, Retrieved from http:/ /indy.cs.concordia.ca/auto/ 
Billings, L., et al. (2007). Instabilities in multiserotype disease models with antibody-dependent enhancement. Journal of Theoretical Biology, 246, 18-27, ISSN 0022-5193.

Centers for Disease Control and Prevention. (2011). Dengue. Retrieved from http://www.cdc.gov/dengue/

Chareonsook, O.et al. (1999). Changing epidemiology of dengue hemorrhagic fever in Thailand. Epidemiol. Infect., 122, 161-166, ISSN 0950-2688.

Dejnirattisai, W. et al. (2010). Cross-Reacting Antibodies Enhance Dengue Virus Infection in Humans. Science, 328, 745-748, ISSN 0036-8075.

Favier, C., et al. (2005). Influence of spatial heterogeneity on an emerging infectious disease: the case of dengue epidemics. Proc. Biol. Sci., 272, 1171-7, ISSN 0962-8452.

Ferguson, N., Anderson, R. and Gupta, S. (1999). The effect of antibody-dependent enhancement on the transmission dynamics and persistence of multiple-strain pathogens. Proc. Natl. Acad. Sci. USA, 96, 790-94, ISSN 0027-8424.

Fischer, D. B. \& Halstead, S. B. (1970). Observations related to pathogenesis of dengue hemorrhagic fever. V. Examination of age specific sequential infection rates using a mathematical model. J. Biol. Med., 42, 329-49.

Gillespie, D.T. (1976). A general method for numerically simulating the stochastic time evolution of coupled chemical reactions. Journal of Computational Physics, 22, 403-434, ISSN 0021-9991.

Gillespie, D.T. (1978). Monte Carlo simulation of random walks with residence time dependent transition probability rates. Journal of Computational Physics, 28, 395-407, ISSN 0021-9991.

Gubler, J. D., Suharyono, W., Tan, R., Abidin, M. and Sie, A. (1981). Viraemia in patients with naturally acquired dengue infection. Bull. World Health Organ., 59, 623-630, ISSN 0042-9686.

Gubler D. J., (2002). Epidemic dengue/dengue hemorrhagic fever as a public health, social and economic problem in the 21st century. Trends in Microbiology, 10, 100-103, ISSN: 0966-842X.

Guzmán, M.G. et al. (2000). Epidemiologic Studies on Dengue in Santiago de Cuba, 1997. Am. J. Epidemiol., 152, 793-799, ISSN 0002-9262.

Guzmán, M.G. et al. (2010). Dengue: a continuing global threat. Nature Reviews Microbiology, 8, S7-S16, ISSN : 1740-1526.

Halstead S. B., et al. (1969). Dengue and chikungunya virus infection in man in Thailand, 1962-1964. V. Epidemiologic observations outside Bangkok. Am. J. Trop. Med. Hyg. 18, 1022-33, ISSN: 0002-9637.

Halstead, S.B. (1982). Immune enhancement of viral infection. Progress in Allergy, 31, 301-364,ISSN 0079-6034.

Halstead, S. B. (1994). Antibody-dependent Enhancement of Infection: A Mechanism for Indirect Virus Entry into Cells. Cellular Receptors for Animal Viruses, 28, Chapter 25, 493-516, ISBN 0-87969-429-7. (Cold Spring Harbor Laboratory Press).

Halstead, S.B. (2003). Neutralization and antibody-dependent enhancement of dengue viruses. Advances in Virus Research, 60, 421-467, ISSN 0065-3527.

He, D., Ionides, E. L., King, A. A. (2010). Plug-and-play inference for disease dynamics: measles in large and small populations as a case study. J. R. Soc. Interface, 7, 271-283, ISSN 1742-5689. 
Holzfuss, J. \& Lauterborn, W. (1989). Liapunov exponents from a time series of acoustic chaos. Physical Review A, 39, 2146-2152, ISSN 1943-2879.

Holzfuss, J. \& Parlitz, U. (1991). Lyapunov exponents from time series. Lecture Notes in Mathematics, 1486, 263-270, ISSN: 0075-8434.

Ionides, E., Breto, C., \& King, A. A. (2006). Inference for nonlinear dynamical systems. Proc. Natl. Acad. Sci. USA, 103, 18438-18443, ISSN 0027-8424.

Lourenço, J. \& Recker, M. (2010). Viral and Epidemiological Determinants of the Invasion Dynamics of Novel Dengue Genotypes. PLoS Negl. Trop. Dis., 4, e894, ISSN 1935-2735.

Mackenzie, J. S., Gubler, D. J. \& Petersen, L. R. (2004). Emerging flaviviruses: the spread and resurgence of Japanese encephalitis, West Nile and dengue viruses. Nature Medicine Review, 12, S98-S109, ISSN : 1078-8956.

Massad, E.,et al. (2008). Scale-free network of a dengue epidemic. Applied Mathematics and Computation, 195, 376-381, ISSN: 0096-3003.

Matheus, S. et al. (2005). Discrimination between Primary and Secondary Dengue Virus Infection by an Immunoglobulin G Aviditnoy Test Using a Single Acute-Phase Serum Sample. Journal of Clinical Microbiology, 43, 2793-2797, ISSN: 0095-1137.

Mattheij, R. M. M. and Molenaar, J. (1996). Ordinary differential equations in theory and practice., ISBN 0-89871-531-8, (Wiley, Chichester and New York).

Monath T. P., (1994). Dengue: The risk to developed and developing countries. Proc. Natl. Acad. Sci. U.S.A., 91, 2395-2400, ISSN 0027-8424.

Nagao, Y. \& Koelle, K.(2008). Decreases in dengue transmission may act to increase the incidence of dengue hemorrhagic fever. Proc. Natl. Acad. Sci. USA, 105, 2238-2243, ISSN 0027-8424.

Nisalak, A. et al. (2003). Serotype-specific dengue virus circulation and dengue disease in Bangkok, Thailand from 1973 to 1999. Am. J. Trop. Med. Hyg., 68, 191-202, ISSN: 0002-9637.

Ott, E. (1993). Chaos in Dynamical Systems, ISBN 0-52143-799-7. (Cambridge University Press, Cambridge, 2nd edition).

Pediatric Dengue Vaccine Initiative. International Vaccine Institute (IVI). Global Burden of Dengue. Retrieved from http://www.pdvi.org/about_dengue/GBD.asp

Recker, M. et al. (2009). Immunological serotype interactions and their effect on the epidemiological pattern of dengue. Proc. R. Soc. B., 276, 2541-2548, ISSN: 1471-2954.

Rigau-Pérez, J. G., et al. (1998). Dengue and dengue haemorrhagic fever. The Lancet, 352, 971-77, ISSN: 0140-6736.

Rosen, L. et al. (1983). Transovarial transmission of dengue viruses by mosquitoes: A. albopictus and A. aegypti. Am. J. Trop. Med. Hyg., 32, 1108-19, ISSN: 0002-9637.

D. Ruelle. (1989). Chaotic Evolution and Strange Attractors, ISBN 978-0-52136-272-6 (Cambridge University Press, Cambridge).

Pers comm.: Francisco Lemos, Secretaria de Estado de Saúde de Minas Gerais, Brazil; Sônia Diniz, Fundação Ezequiel Dias, Minas Gerais, Brazil and Scott Halstead, Pedriatic Dengue Vaccine Initiative, Maryland, USA.

Schwartz, I. B., et al. (2005). Chaotic desynchronization of multi-strain diseases. Physical Review, E 72, 066201-6, ISSN 1943-2879.

Stephenson, J. R., (2005). Understanding dengue pathogenesis: implications for vaccine design. Bull. World Health Organ., 83, 308-14, ISSN 0042-9686. 
Stollenwerk, N., \& Jansen, V. A. A. (2003). Meningitis, pathogenicity near criticality: the epidemiology of meningococcal disease as a model for accidental pathogens. J. Theor. Biol., 222, 347-359, ISSN 0022-5193.

Stollenwerk, N., Maiden, M.C.J. \& Jansen, V.A.A. (2004). Diversity in pathogenicity can cause outbreaks of menigococcal disease. Proc. Natl. Acad. Sci. USA, 101, 10229-10234, ISSN 0027-8424.

Stollenwerk, N., \& Jansen, V. (2010). Population biology and criticality, ISBN 978-1-84816-401-7. (Imperial College Press, London).

Stollenwerk, N., van Noort, S., Martins, J., Aguiar, M., Hilker, F., Pinto, A. \& Gomes G. (2010). A spatially stochastic epidemic model with partial immunization shows in mean field approximation the reinfection threshold. Journal Of Biological Dynamics, 4, 634-649, ISSN: 1751-3758.

Stone, L., Olinky, R., \& Amit Huppert, A. (2007). Seasonal dynamics of recurrent epidemics. Nature, 446, 533-36, ISSN 0028-0836.

World Population Prospects: The 2008 Revision. Population Database. Retrieved from http:/ / esa.un.org/unpp/index.asp?panel=2

van Kampen, N. G. (1992). Stochastic Processes in Physics and Chemistry, ISBN 978-0-44452-965-7. (North-Holland, Amsterdam).

Vaughn, D. W. (2000). Invited Commentary: Dengue Lessons from Cuba. Am. J. Epidemiol., 152, 800-803, ISSN 0950-2688.

Wearing, H.J. \& Rohani, P. (2006). Ecological and immunological determinants of dengue epidemics Proc. Natl. Acad. Sci. USA , 103, 11802-11807, ISSN 0027-8424.

Weisstein, E. W. (2010). "Kermack-McKendrick Model." From MathWorld - A Wolfram Web Resource. Retrieved from http:/ /mathworld.wolfram.com/Kermack-McKendrickModel.html

Wikipedia contributors. Wikipedia, The Free Encyclopedia. Provinces of Thailand. Retrieved from http://en.wikipedia.org/wiki/Provinces_of_Thailand

World Health Organization. (2009). Dengue and Dengue Hemorrhagic Fever, Fact sheet 117. Retrieved from http://www.who.int/mediacentre/factsheets/fs117/en/

World Health Organization: Health statistics and health information systems (2010). The global burden of disease: 2004 update, ISBN 978-9-24156-371-0. Retrieved from http://www.who.int/healthinfo/global_burden_disease/GBD_report_ 2004update_AnnexA.pdf

World Health Organization - Programs and Projects: Initiative for Vaccine Research (2011). Vector borne infections. Retrieved from http://www.who.int/vaccine_research/diseases/vector/en/index1.html\#virology 


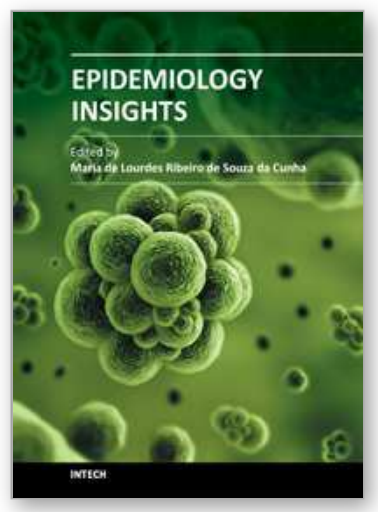

\author{
Epidemiology Insights \\ Edited by Dr. Maria De Lourdes Ribeiro De Souza Da Cunha
}

ISBN 978-953-51-0565-7

Hard cover, 396 pages

Publisher InTech

Published online 20, April, 2012

Published in print edition April, 2012

This book represents an overview on the diverse threads of epidemiological research, brings together the expertise and enthusiasm of an international panel of leading researchers to provide a state-of-the art overview of the field. Topics include the epidemiology of dermatomycoses and Candida spp. infections, the epidemiology molecular of methicillin-resistant Staphylococcus aureus (MRSA) isolated from humans and animals, the epidemiology of varied manifestations neuro-psychiatric, virology and epidemiology, epidemiology of wildlife tuberculosis, epidemiologic approaches to the study of microbial quality of milk and milk products, Cox proportional hazards model, epidemiology of lymphoid malignancy, epidemiology of primary immunodeficiency diseases and genetic epidemiology family-based. Written by experts from around the globe, this book is reading for clinicians, researchers and students, who intend to address these issues.

\title{
How to reference
}

In order to correctly reference this scholarly work, feel free to copy and paste the following:

Maíra Aguiar, Nico Stollenwerk and BobW. Kooi (2012). Modeling Infectious Diseases Dynamics: Dengue Fever, a Case Study, Epidemiology Insights, Dr. Maria De Lourdes Ribeiro De Souza Da Cunha (Ed.), ISBN: 978-953-51-0565-7, InTech, Available from: http://www.intechopen.com/books/epidemiologyinsights/modeling-infectious-diseases-dynamics-dengue-fever-a-case-study

\section{INTECH}

open science | open minds

\author{
InTech Europe \\ University Campus STeP Ri \\ Slavka Krautzeka 83/A \\ 51000 Rijeka, Croatia \\ Phone: +385 (51) 770447 \\ Fax: +385 (51) 686166 \\ www.intechopen.com
}

\author{
InTech China \\ Unit 405, Office Block, Hotel Equatorial Shanghai \\ No.65, Yan An Road (West), Shanghai, 200040, China \\ 中国上海市延安西路65号上海国际贵都大饭店办公楼 405 单元 \\ Phone: +86-21-62489820 \\ Fax: +86-21-62489821
}


(C) 2012 The Author(s). Licensee IntechOpen. This is an open access article distributed under the terms of the Creative Commons Attribution 3.0 License, which permits unrestricted use, distribution, and reproduction in any medium, provided the original work is properly cited. 